\title{
28 Research Square \\ Comparative transcriptome profiling reveals cold stress responsiveness in two contrasting Chinese jujube cultivars
}

\author{
Heying Zhou \\ Beijing Forestry University \\ Ying $\mathrm{He}$ \\ Beijing Forestry University \\ Yongsheng Zhu \\ Wuhan Academy of Agriculture Sciences \\ Meiyu Li \\ Beijing Forestry University

\section{Shuang Song} \\ Beijing Forestry University \\ Wenhao Bo \\ Beijing Forestry University

\section{Yingyue Li} \\ Beijing Forestry University \\ Xiaoming Pang ( $\nabla$ xmpang@163.com ) \\ https://orcid.org/0000-0002-5939-8266
}

\section{Research article}

Keywords: Ziziphus jujuba, 'Dongzao', 'Jinsixiaozao', Freezing stress, RNA-seq, DEGs

Posted Date: April 15th, 2020

DOI: https://doi.org/10.21203/rs.2.21483/v2

License: (c) (i) This work is licensed under a Creative Commons Attribution 4.0 International License. Read Full License

Version of Record: A version of this preprint was published at BMC Plant Biology on May 27th, 2020. See the published version at https://doi.org/10.1186/s12870-020-02450-z. 


\section{Abstract}

Background: Low temperature is a major factor influencing the growth and development of Chinese jujube (Ziziphus jujuba Mill.) in cold winter and spring. Little is known about the molecular mechanisms enabling jujube to cope with different freezing stress conditions. To elucidate the freezing-related molecular changes, we conducted comparative transcriptome analyses from the cold-sensitive cultivar 'Dongzao' and cold-tolerant cultivar 'Jinsixiaozao' using RNASeq.

Results: In our study, more than 20,000 genes were detected at chilling $\left(4^{\circ} \mathrm{C}\right)$ and freezing $\left(-10^{\circ} \mathrm{C},-20^{\circ} \mathrm{C},-30^{\circ} \mathrm{C}\right.$ and $\left.-40^{\circ} \mathrm{C}\right)$ stress between the two cultivars. The numbers of differentially expressed genes (DEGs) between the two cultivars were $1831,2030,1993,1845$ and 2137 under the five treatments. Functional enrichment analysis suggested that the metabolic pathway, response to stimulus and catalytic activity were significantly enriched under stronger freezing stress. Among the DEGs, nine participated in the $\mathrm{Ca}^{2+}$ signal pathway, thirty-two were identified to participate in sucrose metabolism, and others were identified to participate in the regulation of ROS, plant hormones and antifreeze proteins. In addition, important transcription factors (WRKY, AP2/ERF, NAC and $b Z I P$ ) participating in freezing stress were activated under different degrees of freezing stress.

Conclusions: Our research first provides a more comprehensive understanding of DEGs involved in freezing stress at the transcriptome level in two Z. jujuba cultivars with different freezing tolerances. These results may help to elucidate the molecular mechanism of freezing tolerance in jujube and also provides new insights and candidate genes for genetically enhancing freezing stress tolerance.

\section{Background}

Low temperature, as an abiotic stress, not only influences the geographical distribution of many important crops but also negatively impacts the productivity and quality of crops every year [1,2]. Cold stresses are divided into chilling (0$\left.15^{\circ} \mathrm{C}\right)$ and freezing $\left(<0^{\circ} \mathrm{C}\right)$ damage. Freezing stress also has a large impact on the survival and geographical distribution of plants [3]. More than half of the 350,000 plant species on Earth are grown in the tropics and subtropical regions, such as rice, maize, and tomato which are chilling sensitive. However, plants can increase their freezing tolerance by being exposed to chilling, a process known as cold acclimation $[4,5]$.

In previous studies, researchers have revealed that cold exposure results in a variety of alterations in physiology and gene expression patterns, and thousands of genes were even identified that were up- and downregulated in response to cold, including the metabolism of carbohydrates, molecular chaperones, antifreeze proteins, signal transduction (receptor kinase, protein kinase/phosphatase, $\mathrm{Ca}^{2+}$ binding protein) and regulatory proteins [6-10]. In addition, transcription factors could regulate gene expression to improve yields under adverse growing conditions [7,11]. Some TFs (WRKYs and MYBs) are involved in the plant cold response [12-14]. In other species, overexpression of CBF genes in Arabidopsis and other plants conferred enhanced tolerance to cold stresses [15-23]. Additionally, in barley, the overexpression of TaCBF14 and TaCBF15 could enhance freezing tolerance [24]. In addition, both low and freezing temperatures result in oxidative stress due to ROS generation, which includes hydrogen peroxide $\left(\mathrm{H}_{2} \mathrm{O}_{2}\right)$, superoxide anion $\left(\mathrm{O}^{2-}\right)$, and hydroxyl radical (HO) [25-30]. ROS signals can activate redox-responsive proteins, such as transcription factors and protein kinases, such as the mitogen-activated protein kinase (MAPK) cascade [10], and activate of ZAT12 [31]. He et al. [32] found that PeSTZ1 could enhance freezing tolerance through modulation of ROS scavenging by directly regulating PeAPX2 in Populus euphratica.

Chinese jujube (Ziziphus jujuba Mill.) is one of the most important fruit crops in China and has also been introduced to South Korea, the United States and many other countries [33]. In recent years, possibly because global temperature is 
increasing, abnormal extreme weather, such as cold extremes, has been observed to increase all over the world [34,35]. Cold stress also seriously hinders the development of the jujube industry; for example, the quality and productivity of fruits are negatively affected when exposed to low temperature. In terms of woody plants, most studies are focused on physiological and biochemical factors, and little is known about the molecular mechanisms of freezing tolerance [3637]. Saadati et al. [37] analyzed the freezing tolerance of seven olive cultivars by measuring some biochemical and physiological parameters. Nui et al. [38] found some candidate genes potentially involved in freezing tolerance in Prunus persica through high-throughput RNA sequencing. High-throughput RNA sequencing (RNA-seq) technology is useful for the comprehensive analysis of gene functions and metabolic pathways at the transcriptional level. However, it remains unclear how the transcriptome of jujubes responds to freezing stress, and important genes involved in different freezing tolerances are rarely reported. We found that 'Jinsixiaozao' showed a much higher freezing tolerance than 'Dongzao', as indicated by LT50 values, which renders them desirable models for investigating the underlying mechanisms of jujube freezing tolerance (Fig. S1). Thus, we used RNA-seq technology to reveal the molecular response difference between the two cultivars under different degrees of freezing stress. This work provides useful information for understanding the molecular regulation that occurs under freezing tolerance using two cultivars with contrasting freezing tolerance, which provides a rich genetic resource for further research investigating freezing response in jujube and other woody plants.

\section{Results}

\section{Quality analysis and sequence assembly of RNA-seq data}

Total RNA extracted from the xylem of jujube branches was employed to build expression libraries for sequencing by using the Illumina High-Seq sequencing system. We obtained 30 library reads, and each library had approximately 89 million reads. After filtering, clean reads varied from 87 million to 88 million, which occupied on average $97.47 \%$ of the total reads of all libraries (Table 1). In all samples, the Q30 value was over $86 \%$, and the GC content was $43 \%$. The genome map rate accounted for more than $73 \%$, and the gene map rate reached $72.93 \%$ to $76.32 \%$. A total of approximately 20,000 genes were detected to be expressed in each library. In addition, more novel transcripts were detected in both DZD and JSD. Then, regarding alternative splicing, each sample had a similar number of events. Indel, JSD has the maximum mark, significantly more than in DZC. Three biological replicates were collected for each cultivar sample under each temperature. Pearson's correlation coefficients among the three technical replicates were high $(y=0.91-0.98)$, indicating that the sequencing quality enabled further analysis.

\section{DEGs obtained by comparing two cultivars}

To understand the differences between different cultivars in response to freezing stress, gene expression profiles in 'Dongzao' and 'Jinsixiaozao' at the same level of freezing stress were further analyzed though FPKM values. The detailed statistics are shown in Additional file 2. We investigated DEGs specific to each freezing stress by comparing 'Dongzao' to 'Jinsixiaozao'. There were 1831 (885 upregulated genes and 946 downregulated genes), 2030 (979 upregulated genes and 1051 downregulated genes), 1993 (949 upregulated and 1044 downregulated genes), 1845 (923 upregulated and 922 downregulated genes) and 2137 (1158 upregulated and 979 downregulated genes) DEGs were identified at $4{ }^{\circ} \mathrm{C},-10^{\circ} \mathrm{C},-20^{\circ} \mathrm{C},-30^{\circ} \mathrm{C}$ and $-40^{\circ} \mathrm{C}$, respectively, suggesting the responsiveness of these genes to freezing and cold resistance (Fig. 1 and Additional file 3).

In five different degrees of cold stress, as shown in Fig. 2, 854 common DEGs were detected in both cultivars, which may suggest that these DEGs are involved in the process of dealing with low temperature under different low temperatures, and both participate in the same pathways in response to freezing stress. In addition, at $-40^{\circ} \mathrm{C}$, the number 
of unique DEGs (600) was significantly higher than that in the other groups, indicating that these genes may be related to the difference in cold tolerance between 'Dongzao' and 'Jinsixiaozao'.

In this research, total mRNA from branches was used for qRT-PCR validation. As shown in Fig. S2, the genes showed a similar expression pattern between the RNA-seq data and the qRT-PCR results, which indicated that these genes are truly expressed and that the changes at different temperatures are real. Additionally, the results confirmed that the RNA-seq data are highly reliable for further analysis.

\section{Function annotation and enrichment analysis of DEGs}

To understand the functions of the discovered DEGs between the two cultivars, all DEGs were searched against the protein database, followed by Gene Ontology (GO) analysis to assess the putative functions of the genes. Among the GO terms of each treatment, the stronger freezing stress had a larger proportion of DEGs, and the more metabolic processes were modified and changed (Fig. 3). In the comparative analysis of five groups, the DEGs of these GO terms were classified into 'metabolic process', 'cellular process' and 'response to stimulus'; and 'cell', 'cell part' and 'membrane' in cell components; 'catalytic activity', 'binding', 'transporter activity' in molecular function.

In addition, to elucidate the metabolic pathways involved in freezing stress, 20 common metabolic processes were most enriched at the five freezing treatments in the two cultivars (Fig. 4). Among these top pathways, the carbohydrate metabolism pathway, galactose metabolism, fructose and mannose metabolism were associated with many up/downregulated genes. Furthermore, in the comparison of the five groups, there was some pathway enrichment in the stronger freezing stress in 'Jinsixiaozao', such as peroxide of metabolic pathways, which was only significantly enriched at $-30^{\circ} \mathrm{C}$, and the $A B C$ transporter process was enriched at $-40^{\circ} \mathrm{C}$. In brief, many genes of different metabolic pathways were involved in the regulation of freezing stress in jujube, and further study on the differential expression pattern in these pathways has great significance for revealing the cold resistance of jujube.

To further analyze the difference in jujube at extreme temperatures, we conducted a comparative analysis of the two cultivars with temperatures of $-30^{\circ} \mathrm{C}$ and $-40^{\circ} \mathrm{C}$. In comparison, we found that more significantly DEGs were found in the stronger freezing stress. The peroxisome pathway was enriched in DZC vs. JSC, and the ABC transporters pathway was enriched in DZD vs. JSD. In addition, 107430353, annotated to the WRKY family, was significantly changed at lower temperatures. In the ABC family, some members (such as 107429561, 107408533, 107404223, 107414655, and 107416457) simultaneously exhibited significant upregulation. The ratio of DEGs related to kinase activity and carbohydrate metabolism also increased under the stronger freezing stress than under weak freezing stress.

\section{Comparison analysis at different degrees of freezing stress in 'Dongzao' and 'Jinsixiaozao'}

To understand the different effects on jujube at different degrees of freezing stress, we also compared four freezing stresses in 'Dongzao' and 'Jinsixiaozao'. In 'Dongzao', DZCK was used as a control, and 1291 DEGs were identified at different degrees of freezing stress, although only 55 common DEGs were identified in all freezing stresses (Fig. 5, Fig. S3 and Additional file 4). Then, these DEGs were further screened and 327 significant DEGs (PPEE<0.05 and |Log2| $\geq 1$ ) were identified. Of these significant DEGs, some genes were related to the $\mathrm{Ca}^{2+}$ signaling pathway, including upregulated gene $(107413690)$ and downregulated genes $(107404917,107423107,107425924,107417689$, $107421107,107422102,107430853$ and 107424939). Some DEGs related to sucrose metabolism were classified into biological processes among the four groups, including 7 upregulated and 25 downregulated. Furthermore, regarding transcription factors, through the analysis of DEGs, we found that the genes belonging to the NAC, WRKY, MYB, $A P 2 / E R F$ and $b H L H$ families all showed a significant up- or downregulation. A gene (107424282) homologous to the grape $M Y B$ transcription factor is significantly downregulated under cold stress, suggesting that it may negatively regulate the cold resistance of jujube trees. In addition, the NAC transcription factor (107435293) had a higher fold 
change at $-30^{\circ} \mathrm{C}$, indicating that this transcription factor may regulate the cold resistance of jujube trees in response to freezing stress.

In the comparison of four groups of 'Jinsixiaozao', JSCK as a control, a total of 1668 DEGs were identified (Fig. 5, Fig. S4 and Additional file 5), and 86 common DEGs were present in all comparisons. Then we focused analysis on the significant DEGs (PPEE $<0.05$ and $\mid$ Log2 $\mid \geq 1$ ). A total of 71 significantly DEGs were identified at $-10^{\circ} \mathrm{C}, 39$ genes were upregulated and 32 were downregulated. In the case of $-20^{\circ} \mathrm{C}, 108 \mathrm{DEGs}$ were identified, including 31 upregulated genes and 77 downregulated genes. With decreasing temperature, 31 genes were upregulated and 70 genes were downregulated at, $-30^{\circ} \mathrm{C}$. At $-40^{\circ} \mathrm{C}$, only 43 genes had larger changes. Similar to the results of 'Dongzao', many genes associated with abiotic stress have been annotated in 'Jinsixiaozao', such as sucrose metabolism and the $\mathrm{Ca}^{2+}$ signal pathway. Interestingly, among these DEGs, 107422102 was significantly downregulated at $-10^{\circ} \mathrm{C}$ in 'Jinsixiaozao', while it was not changed until the temperature dropped to $-40^{\circ} \mathrm{C}$ in 'Dongzao'. This finding may be due to the different regulatory mechanisms of the two cultivars in response to freezing stress, thereby reflecting different cold resistance.

\section{Key transcription factors associated with freezing stress}

In this study, we further analyzed the expression of key transcription factors (AP2/ERF, WRKY, NAC and bZIP), which have been reported to be involved in freezing stress. All FPKM values of these genes are listed in Additional file 6 . In all libraries, we identified a total of 28 WRKY members, 52 AP2/ ERF members, 22 NAC members and 15 bZIP members in two cultivars (Fig. 6). Comparison of the expression of these TFs between the two cultivars identified WRKY members; some members were highly expressed in 'Dongzao', and others were highly expressed in 'Jinsixiaozao'. Among the TFs, the expression levels of WRKY04 (107417519), WRKY11 (107414569), and WRKY20 (107419756) in 'Jinsixiaozao' were completely different from those in 'Dongzao'. In the AP2/ERF gene family, three of 52 AP2/ERF members (107418844, 107432807 and 107422868) were upregulated under light cold stress in 'Dongzao' but began to change under the lower temperature 'Jinsixiaozao'. In terms of NAC and bZIP, NAC19 had a higher expression level in DZD than in other samples, while in 'Jinsixiaozao', the expression level was higher at all five temperatures; $b Z I P$ members are generally divided into two expression patterns. The observed expression differences of these transcription factors between the two cultivars suggest that TFs may play important roles in the response to different freezing stresses in jujube.

\section{Key DEGs in the galactose metabolism pathway}

The accumulation of stachyose plays an important role in plant resistance to low temperature stress, and agalactosidase is important in the decomposition process of stachyose. In the comparison of the five groups, the galactose metabolic pathway was significantly enriched under each temperature condition. Thus, we further characterized the identified DEGs involved in the metabolism of galactose. During the metabolism of galactose, some genes associated with the galactose, stachyose, raffinose, and sucrose pathways showed significant differences in coping with freezing stress. As listed in Table S2, among these genes, 107411641 is a key gene in the process of UDPgalactose, which is significantly upregulated under stronger freezing stress, which will enhance the synthesis pathway of stachyose. However, in the pathway of stachyose decomposition into galactose and raffinose, the FPKM value of genes $(107425264,107430302,107414011$ and 107428511) decreased, which reduced the decomposition of stachyose (Fig. S5).

\section{Discussion}

Over the past years, extreme weather has occurred frequently, and the variations in climate have affected the growth and development of jujubes and even caused their death. Multiple responses to cold stress have been reported in many plant species, reflected by variations in transcription or metabolism [39]. Recently, significant advances have been made 
regarding the understanding of plant cold-signaling perception and transduction; however, we are still far from fully understanding the molecular mechanism under cold signal perception and transduction in plants [40-42]. In our study, the comparison of transcriptome profiles between 'Jinsixiaozao' and 'Dongzao' was reported to identify the genes responsible for freezing resistance. We first provided a more comprehensive analysis of changes in important metabolic pathways and transcription factors in jujube. Our research could also provide a reference for other woody plants and facilitate breeding for the future development of improved cold- tolerance in jujube cultivars.

\section{Identification of DEGs in two cultivars}

In two cultivars, some DEGs were investigated and hundreds of freezing-related genes were identified in 30 libraries of jujube branches (approximately 87 million reads in 'Dongzao' and 'Jinsixiaozao'). A total of 362 significantly DEGs (323 DEGs in 'Jinsixiaozao') were found to be differentially expressed in 'Dongzao' at different degrees of freezing stress. Compared with the control, most significant DEGs were downregulated. This result is similar to Kou et al. [43] in potato, which suggested that different cultivars have similar responses to cold resistance. However, this finding is different from that of Niu et al.'s [38] study in Prunus persica suffering from freezing tolerance, in which the number of upregulated genes were greater than that of downregulated genes. Moreover, GO classifications of 'Dongzao' and 'Jinsixiaozao' were compared and enriched in functional categories, mainly 'catalytic activity', 'metabolic process', 'cell part' and 'binding'. KEGG pathway analysis revealed that the principal enriched pathways comprised plant hormone signal transduction, metabolic pathways, ribosome and secondary metabolic pathways. With the data provided by the comprehensive analysis using transcriptomic profiling, the important genes involved in the development and regulation of these freezing stress attributes were characterized, which can further contribute to understanding $Z$. jujuba.

\section{Physiological response-related genes under freezing stress}

Some research shows that plants could increase their tolerance by synthesizing numerous protective substances and proteins under these conditions [44-47]. Raffinose family oligosaccharides (RFOs) are a class of functional oligosaccharides unique to plants, mainly raffinose, stachyose and verbascose. Some studies have found that RFO can improve the ability of plants to cope with adversity $[46,48]$. The more RFOs accumulate, the stronger the ability of plants to be exposed to low temperatures becomes. Galactose and related enzymes are involved in the RFO metabolic pathway [48]. In our study, galactose metabolism was significantly enriched, and relative genes in this pathway were up- or downregulated to cope with cold stress. Among these genes, 107426117 (stachyose synthetase), 107415484 (raffinose synthase) and 107411641 (inositol 3-alpha-galactosyltransferase) were significantly upregulated under the stronger freezing treatment in 'Jinsixiaozao', but some of them did not significantly change under the weak freezing stress. This finding may suggest these genes play a more important role in galactose metabolism in response to cold, especially under stronger freezing stresses. In addition, late embryogenesis abundant protein (LEA) could respond to abiotic stresses such as low temperature and drought [49]. In our study, the upregulation of the LEA gene (107409127) indicates that $\angle E A$ genes are involved in freezing tolerance in 'Jinsixiaozao' $\left(-40^{\circ} \mathrm{C}\right)$. The function of LEA protein in this study should be further studied as a regulatory mechanism of freezing response.

\section{Cold signal transduction related genes under freezing stress}

The signal transduction pathway is known to play crucial roles in the response to freezing stress; among these, $\mathrm{Ca}^{2+}$ is an important second messenger in plants $[50,51]$. The enriched $\mathrm{Ca}^{2+}$ signaling pathway genes play critical roles in signaling pathways related to the response to freezing stress [38]. In our research, 34 DEGs were identified as related to calcium-binding proteins, calmodulin and $\mathrm{Ca}^{2+}$ signals. Among them, the gene (107413690) showed a significant upregulation in 'Dongzao', indicating that these genes play important roles in jujube resistance to freezing stress and the function of these genes should be further studied. 
In addition to $\mathrm{Ca}^{2+}$, ROS also play important roles [52]. On the one hand, they induce gene expression and protein synthesis to protect cells from stress; on the other hand, they induce oxidative stress [53,54]. ROS can also directly stimulate the expression of genes in activating stress-related pathways, such as the MAPK cascade pathway, hormone signaling pathway and calcium signaling pathway. In our study, some genes with antioxidant activity were involved in freezing stress. Among these DEGs, six genes (one upregulated gene and five downregulated genes) were involved in redox signaling, such as gene 107403552, which is differentially expressed under four degrees of freezing stress in 'Jinsixiaozao', indicating that they played different roles in cold signal transduction under freezing stress.

\section{Transcriptional regulation-related genes under freezing stress}

Transcription factors (TFs) play important functions in plant development and stress tolerance. Many transcriptomic data have depicted some TFs that govern massive and highly coordinated transcriptional changes under different freezing stresses. Some studies have shown that most cold-regulated transcription factor genes are impaired in response to cold stress in Arabidopsis [55-57]. Many studies have shown that some transcription factors play important roles in plant freezing tolerance [58-60]. For example, the $A P 2 / E R F$ factors $R A P 2.1$ and $R A P 2.6$ and the $\mathrm{C}_{2} \mathrm{H}_{2}$-type zinc finger STZI ZAT10 belong to the CBF regulon [61-62]. Furthermore, NAC was also shown to enhance expression during loquat fruit chilling; however, it could not bind directly to promoters of lignin biosynthesis genes [63]. Many MYB-type transcription factors, such as AtMYB85 [64] and AtMYB88 [65,66], increase cold hardiness by CBF-dependent and CBFindependent pathways in apple. In other species, OSMYB4 transgenic lines of Arabidopsis exhibited improved chilling and freezing tolerance with a dwarf phenotype [67]. In this study, the expression patterns of the AP2/ ERF, WRKY, NAC and $b Z I P$ gene families were analyzed, and we found that different members play different roles in the two cultivars. For example, in the AP2/ ERF family, different members of the family are involved in the response to low temperatures. Among these genes, 107414104 expression showed significant changes at $-10^{\circ} \mathrm{C}$; genes 107432697 and 107433390 were at $-20^{\circ} \mathrm{C} ; 107426263$ and 107433390 were at $-30^{\circ} \mathrm{C}$; however, 107414104,107432697 and 107433390 changed significantly at the lowest temperatures. Similarly, other TF members are generally divided into two expression patterns. These differences suggest that the two cultivars have different responses to cold stress, even for different degrees of freezing stress. We did not go further to verify the function of these genes but listed the significant differences in this work. Thus, the emphasis of future research could focus on the role of these genes in metabolic pathways and understand the regulatory mechanism of genes.

In addition, some studies have found that alternative splicing (AS) events are important posttranscriptional regulators and are required for reprogramming gene expression under environmental stress. Previous studies showed that the number of AS events was significantly higher compared to control plants when plants were exposed to different stresses, especially low temperatures [68]. To analyze the variation in AS under cold stress, in this study, we detected that all genes containing introns were alternately spliced under cold stress (Fig. S6). On the one hand, compared with the control, the number of AS events increases slightly as the temperature drops, and we hypothesize that plants can fine-tune gene expression by AS events in response to temperature changes. On the other hand, most AS genes were not differentially regulated by stress, suggesting that AS may represent an independent gene regulatory layer that responds to cold stress.

\section{Posttranslational regulation-related genes under freezing stress}

In addition, posttranslational modifications are also important for plant cold responses. Dong et al. [69] found that HOS1 can degrade ICE1 and negatively regulate CBFs and downstream cold response genes by ubiquitination under low temperature stress. In this study, the homologous gene (107429115) was found to be significantly upregulated. In addition, with the enhancement of freezing stress, the pathways of phenylpropanoid biosynthesis and flavonoid biosynthesis were also strengthened. Among the significantly DEGs, genes (107429115 and 107415362) are glycosyl- 
transferase-related genes, and the former is significantly upregulated at $-40^{\circ} \mathrm{C}$, and the latter is downregulated, which may mean that they are positively and negatively regulated factors in the process of regulating the cold resistance of jujube, which are involved in different regulatory signaling pathways. Moreover, MLO proteins are a unique protein family in plants, and the expression of many MLO genes is affected by various biotic and abiotic stresses [70]. There is a calmodulin binding region at the C-terminus of the MLO protein, which allows the MLO protein to bind to calmodulin as a second messenger to transmit signals to the downstream pathway. The CaM protein binds to the CaMBD of the MLO protein to increase the activity of the MLO protein. In our data, we found that the gene 107409553 encoding the MLO protein showed a significant upregulation when 'Jinsixiaozao' was at $-40^{\circ} \mathrm{C}$ but showed downregulation in 'Dongzao'. This finding may indicate that the genes regulated by calcium are varietally different between the two cultivars in response to freezing stress.

Some studies have shown that cold stress can restrict plant growth and development at different levels, for example the cell and tissue levels. Roots and branches are both the tissues that predominantly perceive and transduce cold signal in cold stress responses [38, 71-72]. Chilling stress induces DNA damage in root stem cells, and prevents the further division of columella stem cells, which improves the root's ability to overcome cold stress [71]. The crucial coldsignaling regulators such as OST1 and BTF3s are localized to the vasculature in roots and leaves, which means that the vascular tissue of roots might be an important site for plant responses to cold stress [42, 72].

In this study, we comprehensively analyzed the differences in jujube branches at the tissue level in response to cold stress at the transcription level. Further studies showed that some candidate genes are involved in the response to freezing stress, which provided valuable information and genetic resources for improving cold stress in jujube. In addition, we found some significant DEGs under higher degrees of freezing stress, which may suggest that the two cultivars have different responses to different degrees of freezing stress in the metabolic pathway and biosynthesis of galactose. Jujubes sense freezing stress through membrane rigidification and cellular changes, leading to an influx of $\mathrm{Ca}^{2+}$, reactive oxygen species (ROS) and phytohormone changed. The signaling pathways activate protein kinases and changed various downstream transcription factors family proteins, such as WRKY, AP2/ERF, NAC and bZIP. The activation of TFs triggers the expression of downstream responsive genes to the stress treatment, as well as the induction of biochemical responses leading to freezing tolerance. These results may provide evidence to support that 'Jinsixiaozao' has much higher freezing tolerance than 'Dongzao'. Our understanding of the regulatory mechanisms may provide a reference for further research investigating the role of different tissues in freezing stress and other abiotic stresses.

\section{Conclusions}

Our present study first provides a comprehensive analysis via transcriptome sequencing between two Chinese jujube cultivars ('Dongzao' and 'Jinsixiaozao') under freezing stress. The important genes involved in signal transduction and transcriptional and posttranslational regulation were differentially expressed between these two cultivars. Some different members of the $A P 2 / E R F, W R K Y, N A C$ and $b Z I P$ gene families were significantly up/downregulated in the two cultivars. In addition, some DEGs, such as 107426117 and 107411641, involved in galactose metabolism were significantly upregulated under higher degree freezing treatment in 'Jinsixiaozao' than in 'Dongzao', which further highlights the importance of sugar metabolism involved in freezing tolerance in plants. The present results may explain why 'Jinsixiaozao' has considerably higher freezing tolerance than 'Dongzao' and may elucidate the molecular mechanisms of freezing tolerance. The important DEGs discovered in this study may facilitate further study on freezing tolerance mechanisms in jujube and other woody plants.

\section{Methods}




\section{Plant materials and cold treatment}

The current year's branches of cold-sensitive cultivar 'Dongzao' (DZ) and cold-tolerant cultivar 'Jinsixiaozao' (JS) were collected from the National Key Base for Improved Chinese Jujube Cultivar (Cangzhou, China). The collected branches were placed at $4^{\circ} \mathrm{C}$ for $10 \mathrm{~h}$ and used as controls (DZCK and JSCK), the others were treated and maintain $10 \mathrm{~h}$ at the freezing temperature: $-10^{\circ} \mathrm{C}$ (DZA and JSA), $-20^{\circ} \mathrm{C}$ (DZB and JSB), $-30^{\circ} \mathrm{C}$ (DZC and JSC) and $-40^{\circ} \mathrm{C}$ (DZD and JSD). Then the xylem of the branches was collected and snap-frozen in liquid nitrogen. Three replicates were used for each treatment.

\section{RNA-seq library construction and sequencing}

Total RNA were extracted from the 30 samples using the Plant RNA Kit (Omega) according to the manufacturer's instructions. Three biologic replicates for each treatment were used for analyzing transcriptome. The library products were ready for sequencing analysis using an Illumina Genome Analyzer at Beijing Genomics Institute (Shenzhen, China) in 2018. Raw data of fastq format were firstly processed through in-house perl scripts. In this step, clean data were obtained by removing reads containing adapter, reads containing ploy-N and low quality reads from raw data. Q20, Q30 and GC content the clean data were calculated, and all the downstream analyses were based on the clean data with high quality. Reference genome and gene model annotation files were based on Ziziphus jujuba (assembly ZizJuj_1.1) on NCBI. The Z-score method using the p-value as a statistical significance index [73] was applied to identify differentially expressed genes.

\section{Identification of DEGs, GO and KEGG enrichment analysis}

A cluster analysis was performed according to Eisen et al. [74]: the log2 of TPM for each gene was used for the hierarchical clustering analysis. Gene Ontology (GO) categorization was classified into molecular function, biological process, and cellular component. We used KOBAS software to test the statistical enrichment of differential expression genes in KEGG pathways (http://www.genome.jp/kegg/). Four types of AS events including exon skipping, intron retention, alternative $5^{\prime}$ splice site and alternative $3^{\prime}$ splice site were identified using TopHat[75].

\section{Real-time quantitative PCR}

Six randomly selected DEGs were chosen for validation by real-time quantitative PCR (qRT-PCR). The sequences of the primer pairs (designed using Beacon Designer 7.2) were listed in Table S1. Real-time PCR was performed in the presence of TB Green Premix Ex Taq II (Tli RNaseH Plus) (TaKaRa, Dalian, China) and read on an IQ5 real-time PCR instrument (Bio-Rad, Hercules, CA, USA). Each reaction was carried out in a $20 \mu \mathrm{L}$ volume consisting of $10 \mu \mathrm{L}$ of TB Green Premix Ex Taq II, $7.0 \mu \mathrm{L}$ of $\mathrm{ddH}_{2} \mathrm{O}, 2 \mu \mathrm{L}$ template $\left(1 \mu \mathrm{L}\right.$ of the generated first-strand cDNA diluted by $9 \mu \mathrm{L}$ dd $\left.\mathrm{H}_{2} \mathrm{O}\right)$ and $0.5 \mu \mathrm{L}$ of each primer. The following program was used for qRT-PCR: $95^{\circ} \mathrm{C}$ for $10 \mathrm{~min}$ followed by 40 cycles at $95^{\circ} \mathrm{C}$ for $20 \mathrm{sec}, 55^{\circ} \mathrm{C}$ for $20 \mathrm{sec}$, and $72^{\circ} \mathrm{C}$ for $20 \mathrm{sec}$. The baseline and threshold cycles (Ct) were automatically determined by the system's software. All the experiments were performed in biological triplicate. Transcript levels were normalized against the average expression of the Zjactin gene (GenBank: EU916201).

\section{Abbreviations}

DEGs: Differentially Expressed Genes; qRT-PCR: Quantitative real-time PCR; TF: Transcription Factor; MAPK: MitogenActivated Protein Kinase; FPKM: Fragments Per Kilobase Per Million Mapped Reads; AS: Alternative Splicing

\section{Declarations}




\section{Ethics approval and consent to participate}

Not applicable.

\section{Consent for publication}

Not applicable.

\section{Availability of data and materials}

The datasets used and/or analysed have been deposited in NCBI under BioProject ID: PRJNA624182.

\section{Competing interests}

The authors declare that they have no competing interests.

\section{Funding}

This work was financially supported by the National Key Research and Development Program of China (2018YFD1000607), the National Natural Science Foundation of China (31372019), the Beijing Forestry University (2018BLRD). The funding bodies were not involved in the design of the study, collection, analysis, and interpretation of data, and in writing the manuscript.

\section{Authors' contributions}

XMP and HYZ conceived and designed research; HYZ and YH conducted experiments; HYZ and MYL collected and analyzed the data. HYZ wrote the manuscript; SS and YSZ provided valuable suggestions on the manuscript; XMP, WHB and YYL revised the manuscript; XMP obtained funding and is responsible for this article. All authors read and approved the manuscript.

\section{Acknowledgements}

The authors thank the National Key Base for Improved Chinese Jujube Cultivar (Cangzhou, China) for providing the plant materials.

\section{Author details}

${ }^{1}$ Beijing Advanced Innovation Center for Tree Breeding by Molecular Design, National Engineering Laboratory for Tree Breeding, Key Laboratory of Genetics and Breeding in Forest Trees and Ornamental Plants, Ministry of Education, College of Biological Sciences and Biotechnology, Beijing Forestry University, Beijing (100083), China. ${ }^{2}$ Institute of Crop, Wuhan Academy of Agricultural Sciences, Wuhan (430074), China

\section{References}

1. Zhu JK. Abiotic stress signaling and responses in plants. Cell.2016;167:313-324.

2. Guo XY, Liu DF, Chong K. Cold signaling in plants: Insights into mechanisms and regulation. J Integrative Plant Biology.2018;60,9:745-756.

3. Jan N, Andrabi KI. Cold resistance in plants: a mystery unresolved. Electron. J Biotechnol. 2009;12:14-15.

4. Jia YX, Ding YL, Shi YT, Zhang XY, Gong ZZ, Yang SH. The cbfs triple mutants reveal the essential functions of CBFs in cold acclimation and allow the definition of CBF regulons in Arabidopsis. New Phytol.2016;212:345-353. 
5. Chinnusamy V, Zhu J, Zhu JK. Cold stress regulation of gene expression in plants Trends. Plant Sci.2007;12:444451.

6. Thomashow MF. Plant cold acclimation: freezing tolerance genes and regulatory mechanisms. Annu Rev Plant Physiol Plant Mol Biol.1999;50:571-599.

7. Gilmour SJ, Fowler SG, Thomashow MF. Arabidopsis transcriptional activators CBF1, CBF2, and CBF3 have matching functional activities Plant Mol Biol.2004;54:767-781.

8. Chinnusamy V, Schumaker K, Zhu JK. Molecular genetic perspectives on cross-talk and specificity in abiotic stress signalling in plants. J Exp Bot.2004;55:225-236.

9. Min K, Chen KT, Arora R. A metabolomics study of ascorbic acid-induced in situ freezing tolerance in spinach (Spinacia oleracea L.). Plant Direct.2020;4(2):e00202.

10. Colcombet, J, Hirt, H. Arabidopsis MAPKs: a complex signalling network involved in multiple biological processes. Biochem J.2008;413:217-226.

11. Winfield M, Lu C, Wilson I, Coghill J, Edwards K. Plant responses to cold: transcriptome analysis of wheat. Plant Biotechnol J.2010;8:749-771.

12. Mare C, Mazzucotelli E, Crosatti C, Francia E, Stanca AM, Cattivelli L. HvWRKY38: a new transcription factor involved in cold- and droughtresponse in barley. Plant Mol Biol.2004;55:399-416.

13. An JP, Li R, Qu FJ, You CX, Wang XF, Hao YJ. R2R3-MYB transcription factor MdMYB23 is involved in the cold tolerance and proanthocyanidin accumulation in apple. Plant J.2018;96:562-577.

14. An JP, Wang XF, Zhang XW, Xu HF, Bi SQ, You CX, Hao YJ. An apple MYB transcription factor regulates cold tolerance and anthocyanin accumulation and undergoes MIEL1-mediated degradation. Plant Biotechnology Journal.2019;1-17.

15. Jiang BC, Shi YT, Zhang XY, Xin XY, Qi LJ, Guo HW, Li JG, Yang SH. PIF3 is a negative regulator of the CBF pathway and freezing tolerance in Arabidopsis. Proc Natl Acad Sci.2017;114:E6695-E6702.

16. Liu ZY, Jia YX, Ding YL, Shi YT, Li Z, Guo Y, Gong ZZ, Yang SH. Plasma membrane CRPK1-mediated phosphorylation of 14-3-3 proteins induces their nuclear import to fine-tune CBF signaling during cold response. Mol Cell.2017;66:1-12.

17. Anna Fiust, Marcin Rapacz. Downregulation of three novel candidate genes is important for freezing tolerance of field and laboratory cold acclimated barley. Journal of Plant Physiology.2019;244

18. Dubouzet JG, Sakuma Y, Ito Y, Kasuga M, Dubouzet EG, Miura S, Seki M, Shinozaki K, Yamaguchi-Shinozaki K. OsDREB genes in rice, Oryza sativa L, encode transcription activators that function in drought-, high-salt- and coldresponsive gene expression. Plant J.2003;33:751-763.

19. Ma Y, Dai X, Xu Y, Luo W, Zheng X, Zeng D, Pan Y, Lin X, Liu H, Zhang D, Xiao J, Guo X, Xu S, Niu Y, Jin J, Zhang H, Xu X, Li L, Wang W, Qian Q, Ge S, Chong K. COLD1 confers chilling tolerance in rice. Cell. 2015;160:1209-1221.

20. Shen YG, Zhang WK, He SJ, Zhang JS, Liu Q, Chen SY. An EREBP/AP2-type protein in Triticum aestivum was a DREbinding transcription factor induced by cold, dehydration and ABA stress. Theor Appl Genet.2003;106:923-930.

21. Zhang JZ, Creelman RA, Zhu JK. From laboratory to field Using information from Arabidopsis to engineer salt, cold, and drought tolerance in crops. Plant Physiol.2004;135:615-621.

22. Pennycooke JC, Cheng H, Roberts SM, Yang Q, Rhee SY, Stockinger EJ. The low temperature-responsive, Solanum CBF1 genes maintain high identity in their upstream regions in a genomic environment undergoing gene duplications, deletions, and rearrangements. Plant Mol Biol.2008;67:483-497.

23. Pennycooke JC, Cheng H, Stockinger EJ. Comparative genomic sequence and expression analyses of Medicago truncatula and alfalfa subspecies falcata COLD-ACCLIMATIONSPECIFIC genes. Plant Physiol.2008;146:1242- 
1254.

24. Soltesz A, Smedley M, Vashegyi I, Galiba G, Harwood W, Vagujfalvi A. Transgenic barley lines prove the involvement of TaCBF14 and TaCBF15 in the cold acclimation process and in frost tolerance. J Exp Bot.2013;64:1849-1862.

25. Halliwell B. Biochemistry of oxidative stress Biochem Soc Trans.2007;35:1147-1150

26. Hung SH, Yu CW, Lin CH. Hydrogen peroxide functions as a stress signal in plants. Bot Bull Acad Sin.2005;46:1-10.

27. Mittler R, Vanderauwera S, Gollery M, Van BF. Reactive oxygen gene network of plants. Trends Plant Sci.2004;9:490-498.

28. Dat, J, Vandenabeele, S, Vranova E, Van MM, Inze D, Van BF. Dual action of the active oxygen species during plant stress responses. Cell Mol Life Sci.2000;57:779-795.

29. Zhu J, Dong CH, Zhu JK. Interplay between cold-responsive gene regulation, metabolism and RNA processing during plant cold acclimation. Curr Opin Plant Biol.2007;10:290-295

30. Knight H, Knight MR. Abiotic stress signalling pathways: specificity and cross-talk. Trends Plant Sci.2001;6:262267.

31. Davletova S, Schlauch K, Coutu J, Mittler R. The zinc-finger protein Zat12 plays a central role in reactive oxygen and abiotic stress signaling in Arabidopsis. Plant Physiol.2005;139:847-856.

32. He F, Li HG, Wang JJ, Su YY, Wang HL, Feng CH, Yang YL, Niu MX, Liu C, Yin WL, Xia XL. PeSTZ1, a C2H2-type zinc finger transcription factor from Populus euphratica, enhances freezing tolerance through modulation of ROS scavenging by directly regulating PeAPX2. Plant Biotechnology Journal. 2019;17,2169-2183.

33. Liu MJ, Wang JR. Fruit scientific research in New China in the past 70 years: Chinese jujube. Journal of Fruit Science.2019;36(10),1369-1381.(in Chinese)

34. Gupta R, Deswal R. Antifreeze proteins enable plants to survive in freezing conditions. J Biosciences.2014;39:931944.

35. Horton DE, Johnson NC, Singh D, Swain DL, Rajaratnam B, Diffenbaugh NS. Contribution of changes in atmospheric circulation patterns to extreme temperature trends. Nature.2015;522:465-469.

36. Nasrabadi M, Ramezanian A, Eshghi S, KamgarHaghighi AA, Vazifeshenas MR, Valero, D. Biochemical changes and winter hardiness in pomegranate (Punica granatum L.) trees grown under deficit irrigation. Scientia Horticulturae.2019;251,39-47.

37. Saadati S, Baninasab B, Mobli M, \& Gholami M. Measurements of freezing tolerance and their relationship with some biochemical and physiological parameters in seven olive cultivars. Acta Physiologiae Plantarum.2019;41, 51.

38. Niu RX, Zhao XM, Wang CB, Wang FL. Transcriptome profiling of Prunus persica branches reveals candidate genes potentially involved in freezing tolerance. Scientia Horticulturae.2019;259.

39. Shi YT, Ding YL, Yang SH. Molecular Regulation of CBF Signaling in Cold Acclimation. Trends in Plant Science.2018;23,7.

40. Hong JH, Savina M, Du J, Devendran A, Ramakanth KK, Tian X, Sim WS, Mironova VV, Xu J. A sacrifice-for-survival mechanism protects root stem cell niche from chilling stress. Cell.2017;170:26.

41. Zhang Z, Li J, Li F, Liu H, Yang W, Chong K, Xu Y. OsMAPK3 phosphorylate OsbHLH002/OsICE1 and inhibits its ubiquitination to activate OsTPP1 and enhances rice chilling tolerance. Dev Cell.2017;43:731-743.

42. Ding YL, Jia Y, Shi YT, Zhang X, Song C, Gong Z, Yang S. OST1-mediated BTF3L phosphorylation positively regulates CBFs during plant cold responses. EMBO J.2018;e98228.

43. Kou S, Chen L, Tu W, Federico S, Wang YM, Liu J, Fernie AR, Song B, Xie CH. The arginine decarboxylase gene ADC1, associated to the putrescine pathway, plays an important role in potato cold-acclimated freezing tolerance as revealed by transcriptome and metabolome analyses. Plant Journal.2018;96:1283-1298.

Page $12 / 20$ 
44. Steponkus PL, Uemura M, Joseph RA, Gilmour SJ, Thomashow MF. Mode of action of the COR15a gene on the freezing tolerance of Arabidopsis thaliana. Proceedings of the National Academy of Sciences.1998;95:1457014575.

45. Kaplan F, Guy CL. b-Amylase induction and the protective role of maltose during temperature shock. Plant Physiol. 2004;135:1674-1684.

46. Kaplan F, Guy CL. RNA interference of Arabidopsis beta-amylase8 prevents maltose accumulation upon cold shock and increases sensitivity of PSII photochemical efficiency to freezing stress. Plant J.2005;44:730-743.

47. Kaplan F, Kopka J, Sung DY, Zhao W, Popp M, Porat R, Guy CL. Transcript and metabolite profiling during cold acclimation of Arabidopsis reveals an intricate relationship of cold-regulated gene expression with modifications in metabolite content. Plant J.2007;50:967-981.

48. Dai, N, Petreikov, M, Portnoy, V, Katzir, N, Pharr, DM, Schaffer, AA. Cloning and expression analysis of a UDPgalactose/glucose pyrophosphorylase from melon fruit provides evidence for the major metabolic pathway of galactose metabolism in raffinose oligosaccharide metabolizing plants. Plant Physiology.2006;142:294-304.

49. Kosovã K, Vitamvas P, Ilja P. Wheat and barley dehydrins under cold, drought, and salinity-what can LEA-II protein tell us about plant stress response? Frontiers in Plant Science.2014;5:343.

50. Guo XY, Liu DF, Chong K. Cold signaling in plants: Insights into mechanisms and regulation. Journal of Integrative Plant Biology.2018;60:745-756.

51. Yang T, Chaudhuri S, Yang L, Du L, Poovaiah BW. A calcium/calmodulin-regulated member of the receptor- like kinase family confers cold tolerance in plants. J Biol Chem.2010;285:7119-7126.

52. Tyystjarvi E. Photoinhibition of photosystem II. International Review of Cell and Mol Biol.2013;300:243-303.

53. Heidarvand L, Maali-Amiri R. Physio-biochemical and proteome analysis of chickpea in early phases of cold stress. J Plant Physiol.2013;170:459-469.

54. Qi JS, Song, CP, Wang BS, Zhou JM, Kangasjarvi, J, Zhu JK, Gong ZZ. Reactive oxygen species signaling and stomatal movement in plant responses to drought stress and pathogen attack. J Integr Plant Biol.2018;60:805826.

55. Lee SC, Lee MY, Kim SJ, Jun SH, An G, Kim SR. Characterization of an abiotic stress inducible dehydrin gene, OsDhn1, in rice (Oryza sativa L). Mol Cells. 2005;19:212-218

56. Lee BH, Henderson DA, Zhu JK. The Arabidopsis cold-responsive transcriptome and its regulation by ICE1. Plant Cell.2005;17:3155-3175.

57. Zhao C, Wang P, Si T, Hsu CC, Wang L, Zayed O, Yu Z, Zhu Y, Dong J, Tao WA, Zhu JK. MAP kinase cascades regulate the cold response by modulating ICE1 protein stability. Dev Cell.2017;43:618-629.

58. Ding YL, Shi YT, Yang SH. Advances and challenges in uncovering cold tolerance regulatory mechanisms in plants. New Phytologist.2019;doi:101111/nph15696.

59. Li H, Ding Y, Shi Y, Zhang X, Zhang S, Gong Z, Yang S. MPK3- and MPK6-mediated ICE1 phosphorylation negatively regulates ICE1 stability and freezing tolerance in Arabidopsis. Dev Cell.2017;43:630-642.

60. An, JP, Li, R, Qu, FJ, You, CX, Wang, XF and Hao, YJ. An apple NAC transcription factor negatively regulates cold tolerance via CBF dependent pathway. J Plant Physiol.2018;221:74-80.

61. Fowler S, Thomashow MF. Arabidopsis transcriptome profiling indicates that multiple regulatory pathways are activated during cold acclimation in addition to the CBF cold response pathway. Plant Cell.2002;14:1675-1690.

62. Vogel JT, Zarka DG, Van BA, Fowler SG, Thomashow MF. Roles of the CBF2 and ZAT12 transcription factors in confi guring the low temperature transcriptome of Arabidopsis. Plant J.2005;41:195-211.

Page $13 / 20$ 
63. Xu Q, Wang WQ, Zeng JK, Zhang J, Grierson D, Li X, Yin XR, Chen KS. A NAC transcription factor, EjNAC1, affects lignifcation of loquat fruit by regulating lignin. Postharvest Biol Technol.2015;102:25-31.

64. Zhong R, Lee C, Zhou J, McCarthy RL, Ye ZH. A battery of transcription factors involved in the regulation of secondary cell wall biosynthesis in Arabidopsis. The Plant Cell.2008;20:2763-2782.

65. Xie Z, Li D, Wang L, Sack FD, Grotewold E. Role of the stomatal development regulators FLP/MYB88 in abiotic stress responses. Plant Journal.2010;64:731-739.

66. Xie YP, Chen PX, Yan Y, Bao C, Li XW, Wang LP, Shen XX, Li HY, Liu XF, Niu CD, Zhu C, Fang N, Shao Y, Zhao T, Yu JT, Zhu JH, Xu LF, Steven N, Ma FW, Guan QM. An atypical R2R3 MYB transcription factor increases cold hardiness by CBF-dependent and CBF-independent pathways in apple. New Phytologist.2018;218:201-218.

67. Vannini C, Locatelli F, Bracale M, Magnani E, Marsoni M, Osnato M, Mattana M, Baldoni E, Coraggio I, Overexpression of the rice Osmyb4 gene increases chilling and freezing tolerance of Arabidopsis thaliana plants. Plant J.2004;37:115-127.

68. James AB, Syed NH, Bordage S, Marshall J, Nimmo GA, Jenkins GI, Herzyk P, Brown JWS, Nimmo HG. Alternative splicing mediates responses of the Arabidopsis circadian clock to temperature changes. Plant Cell.2012;24:961981.

69. Dong CH, Agarwal M, Zhang Y, Xie Q, Zhu JK. The negative regulator of plant cold responses, HOS1, is a RING E3 ligase that mediates the ubiquitination and degradation of ICE1. Proceedings of the National Academy of Sciences.2006;103:8281-8286.

70. Wang WH, He EM, Guo Y, Tong QX, Zheng HL. Chloroplast calcium and ROS signaling networks potentially facilitate the primed state for stomatal closure under multiple stresses. Environmental and Experimental Botany.2016;122:85-93.

71. Jing HH, Savina M, Jing D, Devendran A, Ramakanth KK, Xin T, Wei SS, Mironova VV, Jian X. A sacrifice-for-survival mechanism protects root stem cell niche from chilling stress. Cell.2017;170,102.

72. Mustilli AC, Merlot S, Vavasseur A, Fenzi F, Giraudat J. Arabidopsis OST1 protein kinase mediates the regulation of stomatal aperture by abscisic acid and acts upstream of reactive oxygen species production. Plant Cell.2002;14,3089-3099.

73. Kale AJ, Zonneveld AJ, Benes V, Berg MVD, Koerkamp MG, Albermann K, Strack N, Ruijter JM, Richter A, Dujon B, Ansorge, W, Tabak AHF, Botstein D. Dynamics of gene expression revealed by comparison of serial analysis of gene expression transcript profiles from yeast grown on two different carbon sources. Mol Biol Cell.1999;10,6:18591872.

74. Eisen MB, Spellman PT, Brown PO, Botstein D. Cluster analysis and display of genome-wide expression patterns. Proc Natl Acad Sci.1998;95(25):14863-14868.

75. Trapnell C, Pachter L, Salzberg SL. TopHat: discovering splice junctions with RNA-Seq. Bioinformatics. 2009;25(9):1105-1111

\section{Table}

Table 1 Summary of the RNA-seq data collected from 'Dongzao' and 'Jinsixiaozao'. 


\begin{tabular}{cccccccccccc}
\hline Sample & Raw data & $\begin{array}{c}\text { Clean } \\
\text { reads }\end{array}$ & Q30(\%) & GC(\%) & $\begin{array}{c}\text { Genome } \\
\text { map } \\
\text { Rate }\end{array}$ & $\begin{array}{c}\text { Gene } \\
\text { map } \\
\text { Rate }\end{array}$ & $\begin{array}{c}\text { Expressed } \\
\text { Gene }\end{array}$ & $\begin{array}{c}\text { Expressed } \\
\text { Transcripts }\end{array}$ & $\begin{array}{c}\text { Novel } \\
\text { Transcripts }\end{array}$ & $\begin{array}{c}\text { Alternative } \\
\text { Splicing }\end{array}$ & $\begin{array}{c}\text { Indel } \\
\text { (n) }\end{array}$ \\
\hline DZCK & 89647985 & 87382161 & 86.91 & 44.19 & $72.64 \%$ & $74.96 \%$ & 20411 & 25818 & 988 & 90011 & 4342 \\
\hline DZA & 89648769 & 87565991 & 88.23 & 44.12 & $73.14 \%$ & $75.11 \%$ & 20362 & 25710 & 954 & 87733 & 8628 \\
\hline DZB & 89648625 & 87947768 & 87.87 & 44.33 & $73.84 \%$ & $76.32 \%$ & 20431 & 25881 & 980 & 88983 & 5811 \\
\hline DZC & 89648887 & 87910197 & 88.73 & 44.19 & $73.52 \%$ & $75.50 \%$ & 20192 & 25458 & 924 & 85154 & 3927 \\
\hline DZD & 89649519 & 87899251 & 88.22 & 44.02 & $73.98 \%$ & $73.91 \%$ & 21546 & 26853 & 1066 & 87281 & 4230 \\
\hline JSCK & 89647603 & 87753183 & 87.40 & 43.64 & $73.12 \%$ & $74.68 \%$ & 20416 & 25724 & 983 & 89086 & 5555 \\
\hline JSA & 89648885 & 87822119 & 89.13 & 43.69 & $72.43 \%$ & $74.23 \%$ & 20264 & 25542 & 983 & 91659 & 4705 \\
\hline JSB & 89648594 & 88010731 & 88.92 & 43.91 & $72.85 \%$ & $74.63 \%$ & 20154 & 25425 & 972 & 86901 & 6013 \\
\hline JSC & 89648221 & 87657475 & 87.74 & 43.70 & $72.58 \%$ & $73.31 \%$ & 19871 & 25011 & 963 & 87767 & 5261 \\
\hline JSD & 89647797 & 87561609 & 87.25 & 43.69 & $73.28 \%$ & $72.93 \%$ & 20648 & 26003 & 1051 & 99095 & 6220 \\
\hline
\end{tabular}

DZCK, 'Dongzao' treated at $4{ }^{\circ} \mathrm{C}$; DZA: 'Dongzao' treated at $-10^{\circ} \mathrm{C}$; DZB: 'Dongzao' treated at $-20^{\circ} \mathrm{C}$; DZC: 'Dongzao' treated at $-30^{\circ} \mathrm{C}$; DZD: 'Dongzao' treated at $-40^{\circ} \mathrm{C}$; JSCK: 'Jinsixiaozao' treated at $4{ }^{\circ} \mathrm{C}$; JSA: 'Jinsixiaozao' treated at $-10^{\circ} \mathrm{C}$; JSB: 'Jinsixiaozao' treated at $-20^{\circ} \mathrm{C}$; JSC: 'Jinsixiaozao' treated at $-30^{\circ} \mathrm{C}$; JSD: 'Jinsixiaozao' treated at $-40^{\circ} \mathrm{C}$.

\section{Supplemental Information}

Additional file1: Figure S1-S6 and Table S1-S2. Figure S1, The semi-lethal temperature (LT50, $\left.{ }^{\circ} \mathrm{C}\right)$ of different cultivars after cold acclimation. Figure S2,Validation by qRT-PCR of DEGs isolated from the different samples in 'Dongzao' and 'Jinsixiaozao'. Figure S3, GO analysis of DEGs under different degree cold stress in 'Dongzao'. The $X$ and $Y$ axes correspond to GO terms and the number of DEGs. Figure S4, GO analysis of DEGs under different degree cold stress in 'Jinsixiaozao'. The $X$ and $Y$ axes correspond to $G 0$ terms and the number of DEGs. Figure S5, DEGs in galactose metabolism. Different boxes represent different genes in galactose metabolism. The red boxes represent up-regulated genes, and the green boxes represents down-regulated genes. Figure S6, Alternative Splicing Event and gene number at the same degree cold stress between two cultivars. Table S1, Primers for qRT-PCR. Table S2, DEGs involved in galactose metabolism pathways.

Additional file2 : FPKM values in 'Dongzao' and 'Jinsixiaozao'.

Additional file3 : DEGs between 'Dongzao' and 'Jinsixiaozao' at the same freezing stress.

Additional file4 : DEGs at different degree freezing stress in 'Dongzao'.

Additional file5 : DEGs at different degree freezing stress in 'Jinsixiaozao'.

Additional file6 : FPKM values of Key transcription factors.

\section{Figures}




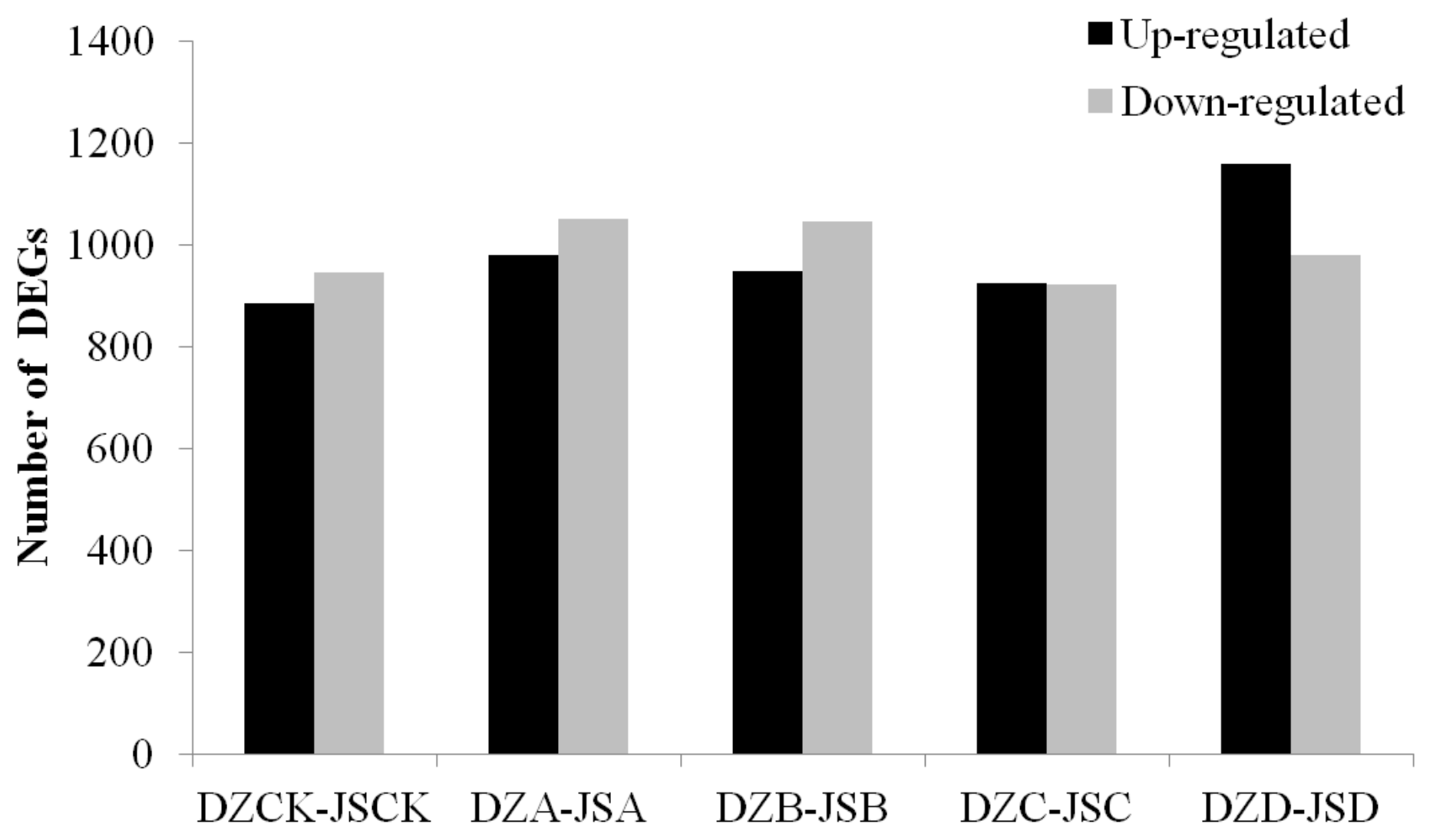

Figure 1

Numbers of DEGs in two cultivars at the same freezing stress. DZCK, 'Dongzao' treated at $4^{\circ} \mathrm{C}$; DZA: 'Dongzao' treated at $-10^{\circ} \mathrm{C}$; DZB: 'Dongzao' treated at $-20^{\circ} \mathrm{C}$; DZC: 'Dongzao' treated at $-30^{\circ} \mathrm{C}$; DZD: 'Dongzao' treated at $-40^{\circ} \mathrm{C}$; JSCK: 'Jinsixiaozao' treated at $4{ }^{\circ} \mathrm{C}$; JSA: 'Jinsixiaozao' treated at $-10^{\circ} \mathrm{C}$; JSB: 'Jinsixiaozao' treated at $-20^{\circ} \mathrm{C}$; JSC: 'Jinsixiaozao' treated at $-30^{\circ} \mathrm{C}$; JSD: 'Jinsixiaozao' treated at $-40^{\circ} \mathrm{C}$. 


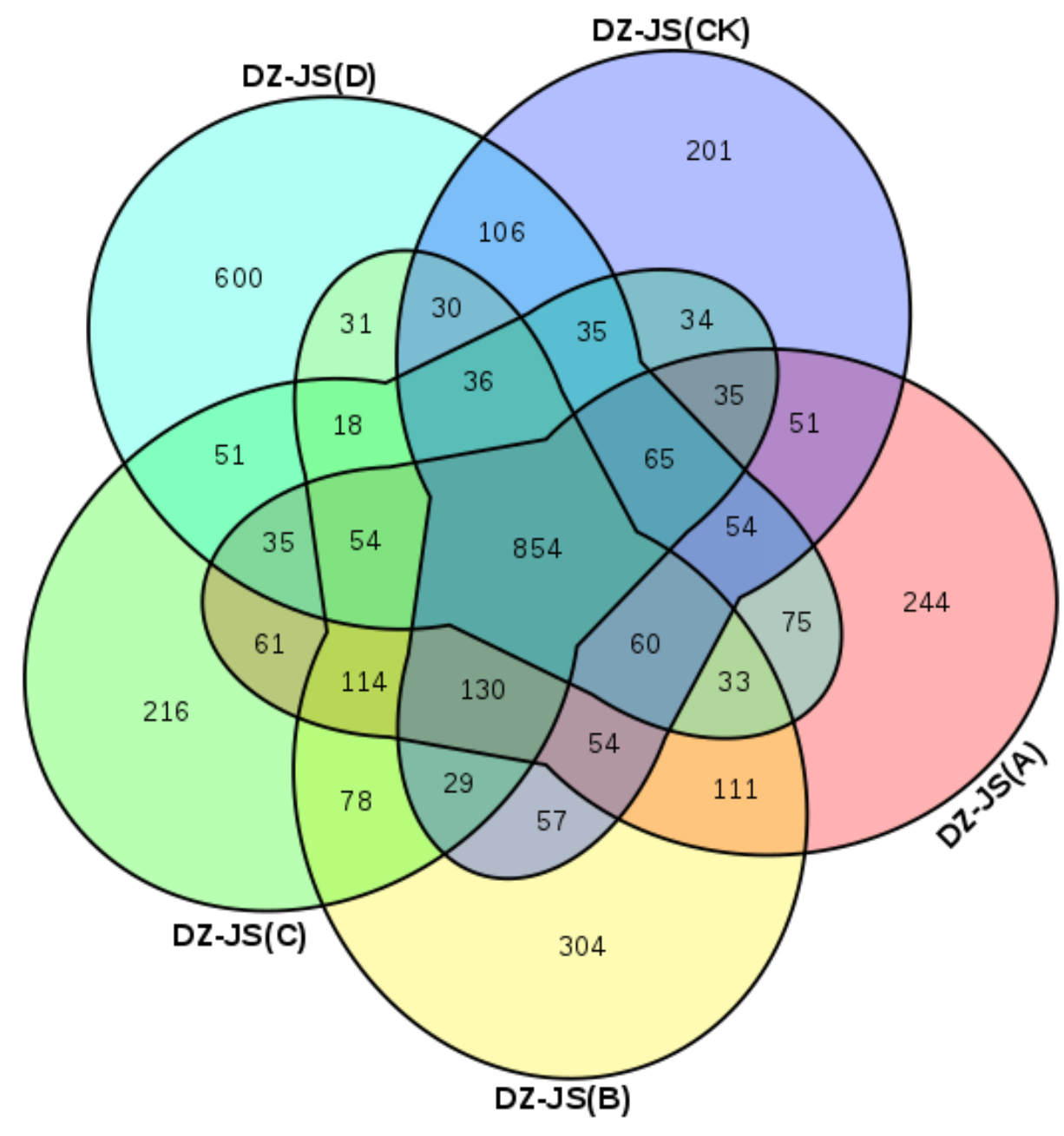

Figure 2

Venn diagram of DEGs under the same freezing stress in 'Dongzao' and 'Jinsixiaozao'. DZ, 'Dongzao'; JS, 'Jinsixiaozao'; $\mathrm{CK}$, treated at $4{ }^{\circ} \mathrm{C} ; \mathrm{A}$, treated at $-10^{\circ} \mathrm{C} ; \mathrm{B}$, treated at $-20^{\circ} \mathrm{C} ; \mathrm{C}$, treated at $-30^{\circ} \mathrm{C} ; \mathrm{D}$, treated at $-40^{\circ} \mathrm{C}$. 


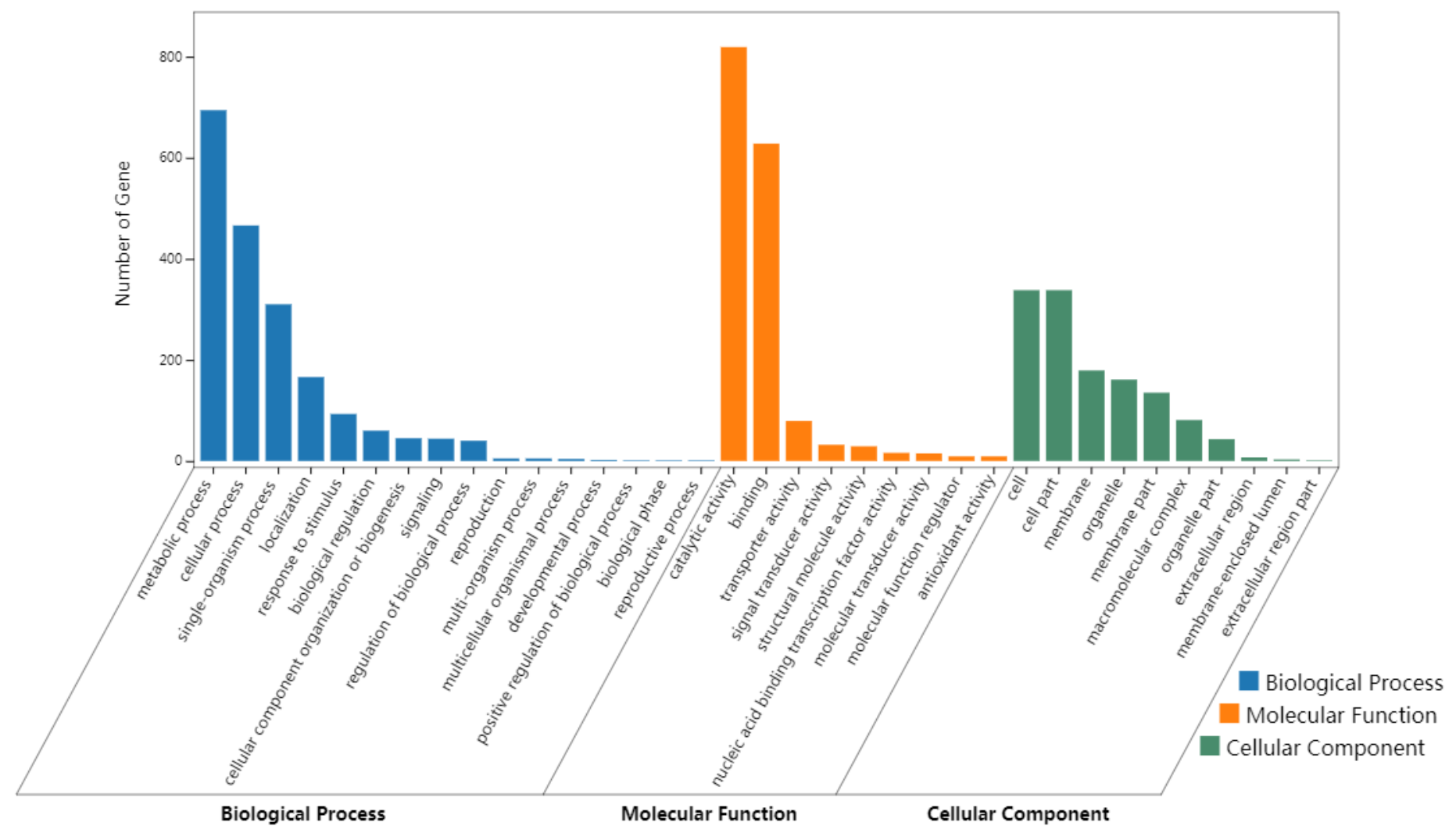

Figure 3

GO analysis of DEGs at the same freezing stress between two cultivars. GO classification of DEGs under cold stress in 'Dongzao' and 'Jinsixiaozao', respectively. The $\mathrm{Y}$ and $\mathrm{X}$ axes correspond to $\mathrm{GO}$ terms and the number of DEGs. 


\section{Top 20 of Pathway Enrichment}

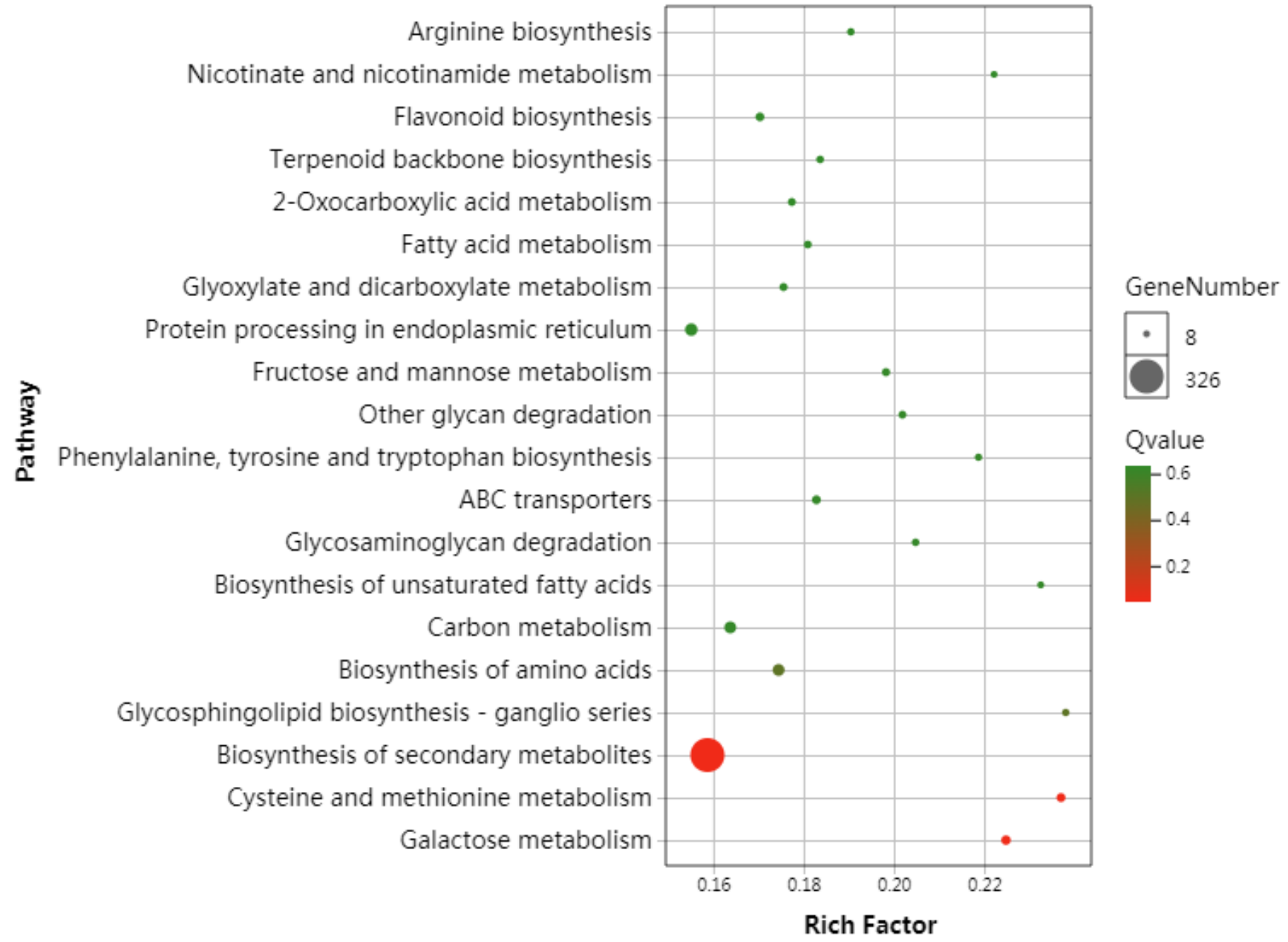

Figure 4

KEGG enrichment analysis of DEGs at the same freezing stress between two cultivars. KEGG pathway classifcation of DEGs under cold stress in 'Dongzao' and 'Jinsixiaozao', respectively. The $Y$ axis corresponds to KEGG pathway, the $X$ axis shows the enrichment ratio between the number of DEGs enriched in a particular pathway. The color of the dot represents q-value, and the size of the dot represents the number of DEGs mapped to the referent pathway.
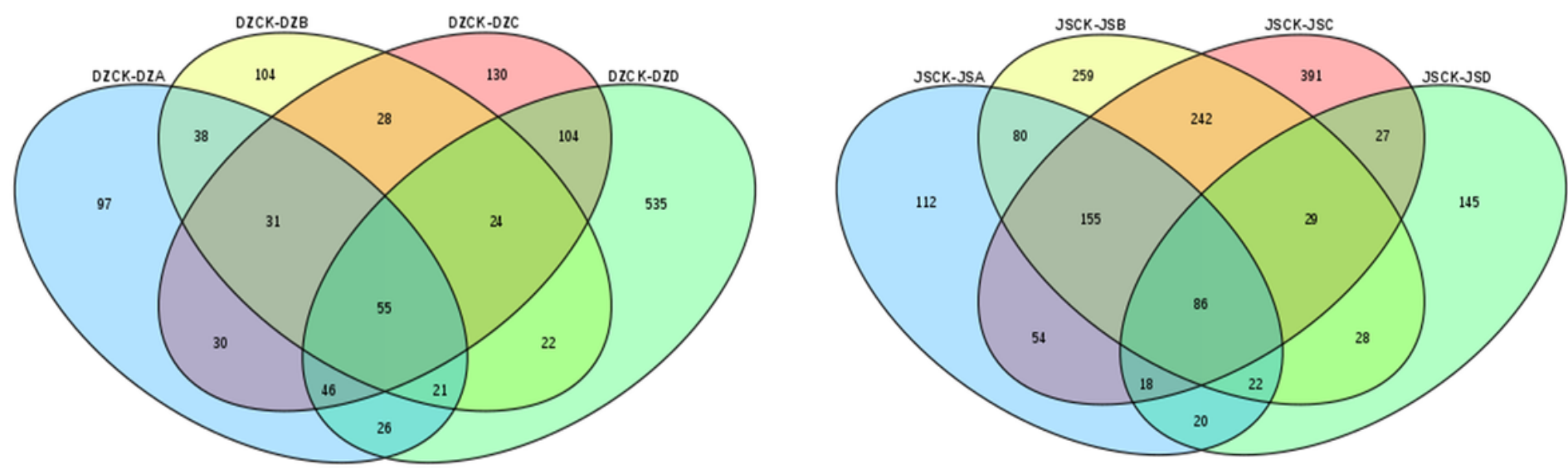
Figure 5

Venn diagram of DEGs in 'Dongzao' (left) and 'Jinsixiaozao' (right). DZCK, 'Dongzao' treated at $4{ }^{\circ} \mathrm{C}$; DZA: 'Dongzao' treated at $-10^{\circ} \mathrm{C}$; DZB: 'Dongzao' treated at $-20^{\circ} \mathrm{C}$; DZC: 'Dongzao' treated at $-30^{\circ} \mathrm{C}$; DZD: 'Dongzao' treated at $-40^{\circ} \mathrm{C}$; JSCK: 'Jinsixiaozao' treated at $4^{\circ} \mathrm{C}$; JSA: 'Jinsixiaozao' treated at $-10^{\circ} \mathrm{C}$; JSB: 'Jinsixiaozao' treated at $-20^{\circ} \mathrm{C}$; JSC: 'Jinsixiaozao' treated at $-30^{\circ} \mathrm{C}$; JSD: 'Jinsixiaozao' treated at $-40^{\circ} \mathrm{C}$.
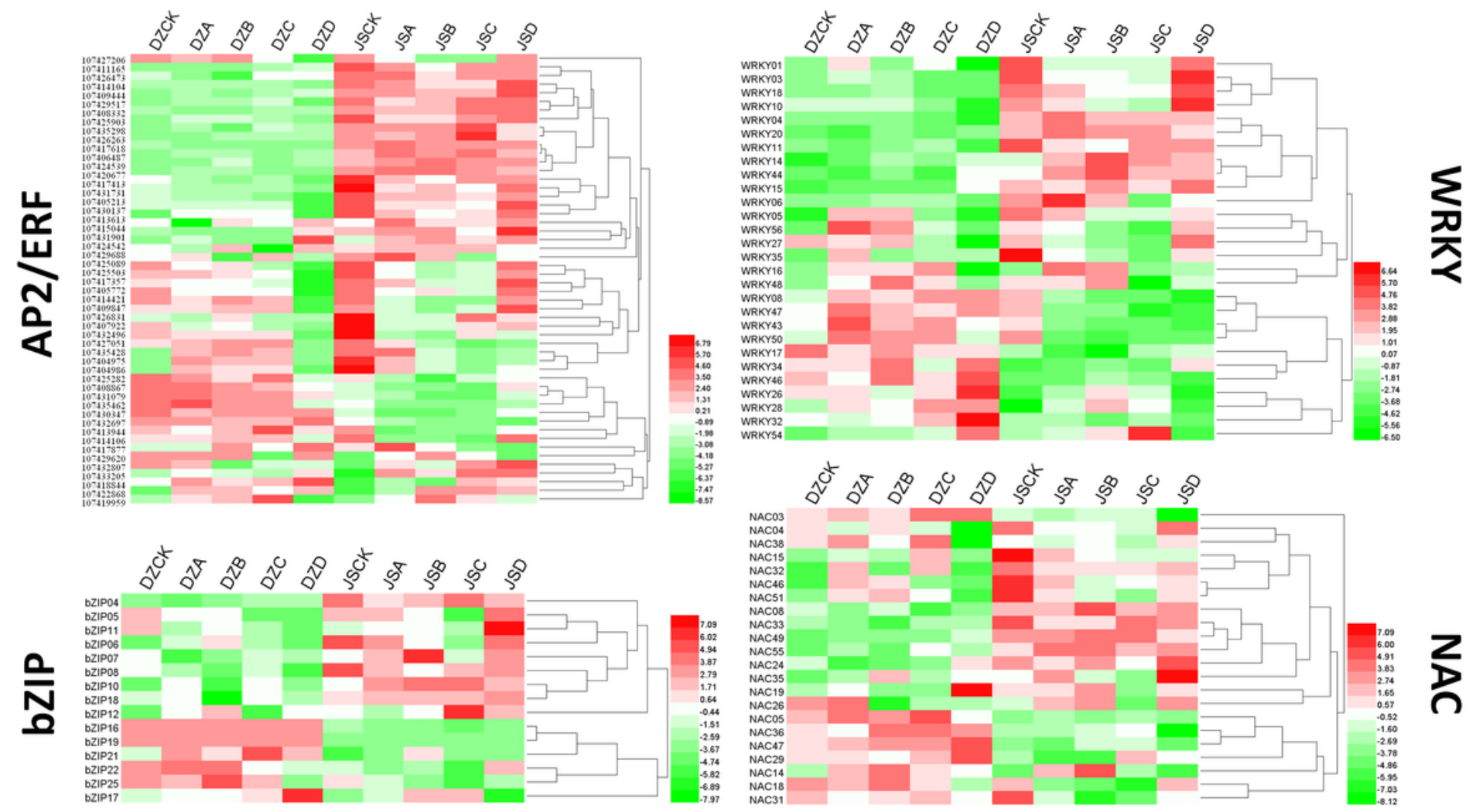

Figure 6

Expression profiles of the jujube AP2/ERF, WRKY, bZIP and NAC genes in two cultivars. DZCK, 'Dongzao' treated at $4{ }^{\circ} \mathrm{C}$; DZA: 'Dongzao' treated at $-10^{\circ} \mathrm{C}$; DZB: 'Dongzao' treated at $-20^{\circ} \mathrm{C}$; DZC: 'Dongzao' treated at $-30^{\circ} \mathrm{C}$; DZD: 'Dongzao' treated at $-40^{\circ} \mathrm{C}$; JSCK: 'Jinsixiaozao' treated at $4{ }^{\circ} \mathrm{C}$; JSA: 'Jinsixiaozao' treated at $-10^{\circ} \mathrm{C}$; JSB: 'Jinsixiaozao' treated at $-20^{\circ} \mathrm{C}$; JSC: 'Jinsixiaozao' treated at $-30^{\circ} \mathrm{C}$; JSD: 'Jinsixiaozao' treated at $-40^{\circ} \mathrm{C}$. Data were normalized based on the mean expression value of each gene in all samples analysed. Red and green boxes indicate high and low expression levels, respectively, for each gene.

\section{Supplementary Files}

This is a list of supplementary files associated with this preprint. Click to download.

- Additionalfile6.xIsx

- Additionalfile3.xIsx

- Additionalfile4.xlsx

- Additionalfile1.docx

- Additionalfile2.xIsx

- Additionalfile5.xlsx 